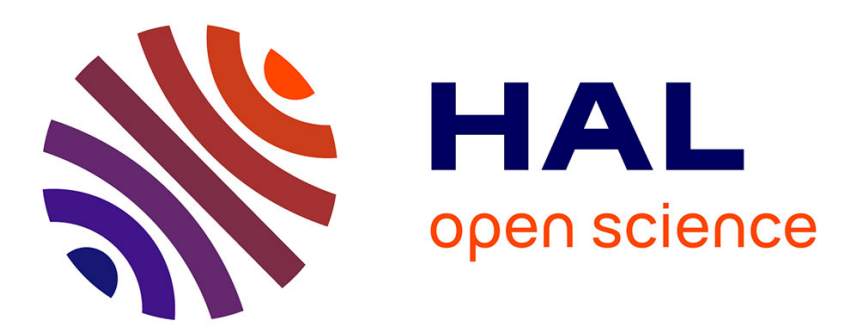

\title{
Measurements of surface elastic torques in liquid crystals : a method to measure elastic constants and anchoring energies
}

S. Faetti, M. Gatti, V. Palleschi

\section{- To cite this version:}

S. Faetti, M. Gatti, V. Palleschi. Measurements of surface elastic torques in liquid crystals : a method to measure elastic constants and anchoring energies. Revue de Physique Appliquée, 1986, 21 (7), pp.451-461. 10.1051/rphysap:01986002107045100 . jpa-00245463

\section{HAL Id: jpa-00245463 https://hal.science/jpa-00245463}

Submitted on 1 Jan 1986

HAL is a multi-disciplinary open access archive for the deposit and dissemination of scientific research documents, whether they are published or not. The documents may come from teaching and research institutions in France or abroad, or from public or private research centers.
L'archive ouverte pluridisciplinaire HAL, est destinée au dépôt et à la diffusion de documents scientifiques de niveau recherche, publiés ou non, émanant des établissements d'enseignement et de recherche français ou étrangers, des laboratoires publics ou privés. 


\title{
Measurements of surface elastic torques in liquid crystals : a method to measure elastic constants and anchoring energies $(*)$
}

\author{
S. Faetti $\left({ }^{+\circ}\right)$, M. Gatti $\left(^{+}\right)$and V. Palleschi $\left({ }^{+}\right)$ \\ $\left(^{+}\right)$Dipartimento di Fisica dell' Universita' di Pisa, 56100 Pisa, Italy \\ ( ${ }^{\circ}$ Gruppo Nazionale di Struttura della Materia del CNR, Piazza Torricelli 2, 56100 Pisa, Italy
}

(Reçu le 23 septembre 1985, révisé les 6 décembre 1985, et 7 avril 1986, accepté le 7 avril 1986)

\begin{abstract}
Résumé. - Le couple exercé par un cristal liquide nématique sur les surfaces est mesuré en utilisant un pendule de torsion. Ce couple est engendré par un champ magnétique qui provoque une distorsion du directeur. Trois géométries différentes sont étudiées. Les constantes élastiques et l'énergie d'ancrage du cristal liquide nématique peuvent être obtenues par la mesure du couple. L'avantage de cette méthode est que la mesure des constantes élastiques est peu sensible aux petites désorientations du directeur près des surfaces et a une valeur finie de l'énergie d'ancrage. Nous avons utilisé cette technique pour la mesure des constantes élastiques $K_{11}$ and $K_{33}$ du cristal liquide 4-pentyl-4'-cyanobiphenyl (5CB). Les résultats de l'expérience sont comparés avec des mesures précédentes.
\end{abstract}

\begin{abstract}
Surface torques exerted by a nematic liquid crystal on a solid plate are investigated. Three different geometries are considered. The torques are generated by applying a uniform magnetic field to the nematic sample and are measured by a torsion pendulum. The elastic constants of the nematic Liquid Crystal and the anchoring energy at the interface can be obtained by this kind of measurements. As a main advantage of this technique, the measurements of the elastic constants are poorly sensitive to small misalignments of the director and are not affected from a finite value of the anchoring energy. Experimental results for the splay and bend elastic constants of the nematic LC 4-pentyl-4'-cyanobiphenyl (5CB) are given and compared with previous experimental results.
\end{abstract}

\section{Introduction.}

Elastic constants of nematic liquid crystals (LC) play an important rôle on the macroscopic properties of these materials. As a consequence of this, a lot of experiments have been performed to measure elastic constants of nematic LC [1-13]. Most of the experiments concern the investigation of director deformations induced by magnetic or electric fields in thin layers of nematic LC [1-8]. Other experiments concern the investigation of the light scattering from an uniformly aligned layer of nematic LC [9-11]. These latter experiments are affected by larger uncertainties. Other methods have been proposed but they do not seem to furnish a sufficient accuracy $[12,13]$. Therefore so far the most reliable values of the elastic constants have been obtained from measurements of the Freedericksz transition in thin nematic layers. In most of these

$\left(^{*}\right)$ Research supported in part by Ministero della Pubblica Istruzione and in part by Consiglio Nazionale delle Ricerche, Italy. experiments the magnetic (or electric) field is applied perpendicular to the director and a director distortion occurs when the field exceeds a threshold value $H_{\mathrm{c}}$. By assuming strong anchoring of the director at the interfaces of the nematic layer one obtains [14]

$$
H_{\mathrm{c}}=\sqrt{\frac{K_{i i}}{\chi_{\alpha}}} \frac{\pi}{d}
$$

where $d$ is the thickness of the layer, $\chi_{\alpha}$ is the anisotropy of the diamagnetic susceptibility and $K_{i i}$ is an elastic constant ( $i=1$ splay, $i=2$ twist, $i=3$ bend). The three values of the index $i$ correspond to three different experimental geometries [14]. Rapini and Papoular [15] investigated the sensitivity of the threshold field to small misalignments of the director and to a finite value of the anchoring energy. They found both these factors reduce the threshold field, in particular a $2^{\circ}$-misalignment of the director at the surfaces gives a reduction of $10 \%$ in the threshold magnetic field. This high sensitivity to the experimental conditions can explain some large discrepancies between different 
measurements of elastic constants. We note, however, that in recent papers [6] Oldano et al. proposed a " modified Freedericksz technique " which avoids the previous error sources and furnishes accurate values of the elastic constants. In the following we will denote the conventional Freedericksz method as "standard " Freedericksz technique and the method of reference [6] as « modified" Freedericksz technique.

In a recent paper Grupp [16] proposed a new experimental method to measure the twist elastic constant of nematic LC. The method consists in measuring the elastic torque exerted by a twisted nematic layer on the surfaces. The two parallel plane glass plates which bound the nematic layer are treated in such a way as to induce a planar orientation of the director. The twist of the director-field is generated by rotating one of the two plates. The main drawback of this method is related to the long measuring time ( $\sim$ a day) due to viscosity effects. In a recent paper [17] we showed that the measuring time can be largely reduced (to a few minutes) using thick nematic samples and generating the twist of the director field by means of a magnetic field.

In this paper we show that the experimental method of reference [17] can be suitably extended to measure the bend and splay elastic constants of nematic LC. Three different geometries are investigated which allow us to measure the three elastic constants $K_{11}$, $K_{22}$ and $K_{33}$. The main advantages and drawbacks of this new experimental technique are discussed. The bend and splay elastic constants of the nematic LC 4-pentyl-4'-cyanobiphenyl (5CB) are measured and the experimental results are compared with previous results obtained by other authors.

\section{Principle of the measurement and experimental apparatus.}

Figure 1 shows schematically the experimental apparatus used to measure surface elastic torques. The nematic LC sample (NLC in Fig. 1) lies on the bottom of a cylindrical glass cell which is thermostated by a water thermal bath $(T)$ with a temperature stability of $10 \mathrm{mK}$. The temperature is measured by means of a linear thermoresistor (TR in Fig. 1). A glass plate $(P)$ is dipped in the nematic sample and is sealed to a vertical glass tube $(Q)$. Three different configurations are investigated ( $i=1,2,3$ in Fig. 2). The glass plate is suspended by a thin quartz wire (W) through the glass tube $\mathrm{Q}$. The torsion pendulum can be rotated around the vertical axis by means of a rotation stage $(R)$ which lies on the top of the cylindrical glass cell. An uniform magnetic field $\mathrm{H}$ ranging from 50 to $8800 \mathrm{G}$ can be applied along the horizontal $x$-axis. When the easy axis of the director on the glass plate is not parallel to the magnetic field, a distortion of the director-field occurs within a thin layer close to the surface (see Fig. 3) (the thickness of this layer is of the order of the

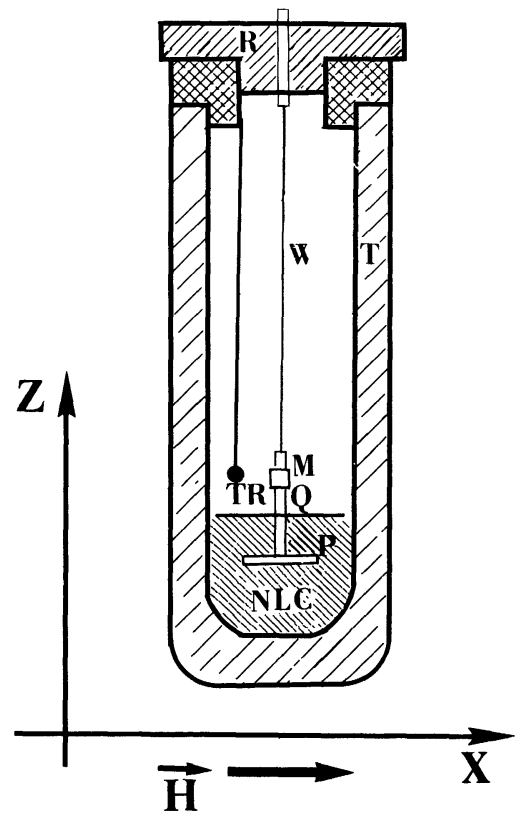

Fig. 1. - Schematic vertical cross section of the experimental apparatus. NIC = nematic sample, $P=$ glass plate (the geometry $i=2$ of Fig. 2 is shown), $\mathrm{Q}=$ vertical thin glass tube, $\mathbf{M}=$ glass plate (used as a mirror to detect the rotation of the torsion pendulum), $\mathrm{W}=$ thin quartz wire $(30 \mu \mathrm{m}), \quad \mathrm{R}=$ rotation stage, $\mathrm{T}=$ thermostatic bath of circulating water, $T R=$ linear thermoresistor, $\mathbf{H}=$ magnetic field.

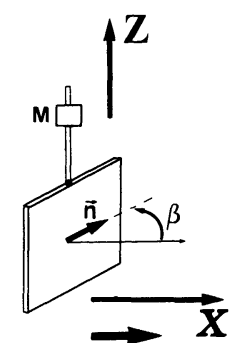

$\overline{\mathbf{H}}$

$i=1$

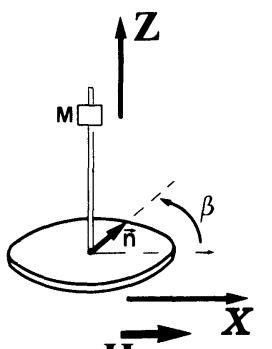

$\overline{\mathrm{H}}$

$i=2$

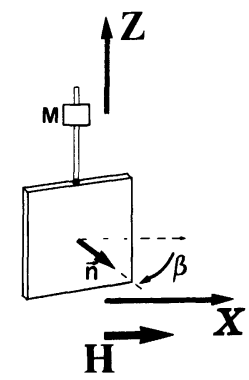

$i=3$
Fig. 2. - The three geometries of the glass plate $P$ of figure 1 and the corresponding director orientation at the surface are shown : $i=1$, the easy axis of the director $\mathbf{n}$ is parallel to the surface of the rectangular glass plate (homogeneous orientation); $i=2$, the easy axis of the director is parallel to the surface of the circular glass plate; $i=3$, The easy axis of the director is orthogonal to the rectangular glass plate. $\beta$ is the angle between the magnetic field and the director at the surface of the glass plate.

magnetic coherence length $\xi=\sqrt{\frac{K_{i i}}{\chi_{\alpha}}} \frac{1}{H}$ and ranges from $2 \mu \mathrm{m}$ to $20 \mu \mathrm{m}$ in our experiment). Under these conditions the nematic sample exerts a torque on the glass plate $P$ and thus, the torsion pendulum rotates. 


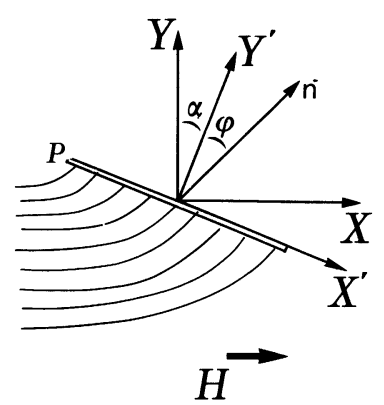

(a)
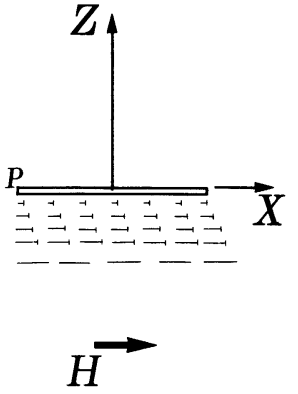

(b)
Fig. 3. - Schematic view of the director distortion near the glass plate. a) Cases $i=1\left(\varphi=-90^{\circ}\right)$ and $i=3\left(\varphi=0^{\circ}\right)$ : horizontal cross section of the glass plate P. $\alpha$ is the angle between the axis $y^{\prime}$ orthogonal to the plate and the $y$-axis orthogonal to the magnetic field, $\varphi$ is the angle between the director at the surface and the $y^{\prime}$-axis. The director-field lines in the presence of the magnetic field are shown schematically. For convenience the director-field has been drawn only in the lower region of the figure. A symmetric distortion of the director occurs in the upper region above the glass plate. b) Case $i=2$ (twist distortion) : vertical cross section of the circular glass plate $P$. The easy axis of the director lies on the surface of the glass plate and makes the angle $\alpha_{0}$ with the $y$-axis.

This rotation is detected from the deflection of a laser beam which is reflected by a small glass plate $M$ sealed to the glass tube $\mathrm{Q}$. The accuracy of the measurement of the rotation angle $\Delta \alpha$ of the torsion pendulum is $10^{-4} \mathrm{rad}$. The restoring torque of the torsion wire is :

$$
\tau=-k \Delta \alpha
$$

where $k$ is the torsion coefficient of the quartz wire which is measured with an accuracy better than $0.5 \%$.

The elastic torque exerted by the nematic LC on the glass plate $\mathbf{P}$ can be calculated by using the elastic theory of LC [14]. Let $S^{\prime}$ be the surface of the glass plate and let $S^{\prime \prime}$ be an ideal closed surface which bounds $S^{\prime}$ and lies far away from this surface where the director is uniformly aligned along the magnetic field. The elastic surface torque on the glass plate is balanced by the magnetic torque acting on the volume bounded by the surfaces $S^{\prime}$ and $S^{\prime \prime}$, i.e.

$$
\tau=\iiint_{\Omega} \chi_{\alpha}(\mathbf{H} \cdot \mathbf{n})(\mathbf{n} \wedge \mathbf{H}) \mathrm{d} V
$$

where $\mathbf{n}$ is the director. The dependence of $\mathbf{n}$ on spatial coordinates is obtained by minimizing the total free energy $\mathfrak{J}$ of the system [14] :

$$
\begin{aligned}
\mathfrak{J} \equiv \iiint\left\{\frac{1}{2}[\right. & K_{11}(\operatorname{div} \mathbf{n})^{2}+K_{22}(\mathbf{n} \cdot \operatorname{rot} \mathbf{n})^{2}+ \\
& \left.\left.+K_{33}(\mathbf{n} \wedge \operatorname{rot} \mathbf{n})^{2}\right]-\frac{\chi_{\alpha}}{2}(\mathbf{H} \cdot \mathbf{n})^{2}\right\} \mathrm{d} V \\
& +\iint_{S^{\prime}} W\left(\varphi_{0}\right) \mathrm{d} \Sigma
\end{aligned}
$$

where $\mathbf{n}$ is the director. $W\left(\varphi_{0}\right)$ is the anchoring energy on the glass plate [14] and $\varphi_{0}$ is the angle between the director and the easy axis at this interface. As a first approximation the anchoring energy is usually assumed to have the simple form [18] :

$$
W\left(\varphi_{0}\right)=W_{0} \sin ^{2}\left(\varphi_{0}\right)
$$

where $W_{0}$ is the anchoring energy coefficient. The Frank-Ericksen elastic constant $K_{24}[19]$ is not included in equation (4) since it does not contribute to the free energy in the case of the two-dimensional director distortions shown in figure 3. Nehring and Saupe [20] extended the Frank-Ericksen theory by introducing a new elastic term proportional to the elastic constant $K_{13}$. This term does not influence the bulk free energy but it modifies the surface free energy. In a recent paper Barbero and Oldanc showed [21] that the presence of this elastic surface terms induces some difficulties in obtaining the correct boundary conditions for the director-field. In particular all previously proposed boundary conditions are incorrect (see references given in reference [21]). Since the mathematical and physical problem of accounting for the effect of the surface-like elastic constant $K_{13}$ has not yet been solved, we here neglect the effect of this latter constant $\left(K_{13}=0\right)$.

In our experiment the thickness $\xi$ of the distorted layer close to the surfaces is much smaller than the other characteristic lengths of the system (thickness of the glass plate $\left(\sim 10^{-2} \mathrm{~cm}\right)$ and average thickness of the nematic sample $(\sim 0.3 \mathrm{~cm})$ ). Furthermore the thickness of the glass plate is so small that boundary effects can be neglected. Therefore the angle $\theta$ which the director makes with the magnetic field can be assumed to depend only on the distance from the glass plate (this distance is denoted by $z$ in the twist geometry $(i=2)$ and by $y^{\prime}$ in the other cases). The angles $\theta$ and $\varphi_{0}$ are obtained by minimizing the free energy (Eq. (4)) with respect to variations of $\theta$ and $\varphi_{0}$. Once $\theta$ is found it can be substituted in equation (3) to obtain the surface elastic torque $\tau$. In the case $i=2$ (twisted geometry of Fig. 3b) one obtains :

$$
\begin{aligned}
& \frac{\tau}{S}=\mp \sqrt{K_{22} \chi_{\alpha}} H \cos \left(\alpha_{0}+\varphi_{0}\right) \\
& \frac{\tau}{S}=-\frac{\partial W\left(\varphi_{0}\right)}{\partial \varphi_{0}}=-W_{0} \sin 2 \varphi_{0}
\end{aligned}
$$

where $\alpha_{0}$ is the angle between the easy axis of the director and the $y$-axis orthogonal to the magnetic field and $S$ is the total area of the two plane surfaces of the glass plate. $\varphi_{0}$ is the angle of the director at the surface with respect to the easy axis. Equation (7) represents the boundary conditions which must be satisfied by the surface director angle $\varphi_{0}$. According to this equation the elastic torque acting on the director at the surface is balanced by the surface anchoring torque $\left(-\frac{\partial W}{\partial \varphi_{0}}\right)$. Note that, for a given value of the torque $\tau$, 
the surface angle $\varphi_{0}$ is almost zero if $W_{0} \gg \sqrt{K_{22} \chi_{\alpha}} \times$ $H \cos (\alpha)$. Therefore for a low enough magnetic field, one can assume $\varphi_{0}=0$ in equation (6) (strong anchoring at the interface). The signs $\mp$ stand for the two cases $0<\alpha_{0}<\pi$ and $-\pi<\alpha_{0}<0$, respectively. Two different director distortions having opposite twists correspond to the two signs in equation (6). If $\alpha_{0}=0$ both these distortions have the same free energy and thus, one expects that alternate domains separated by walls [22] occur giving an almost vanishing torque on the glass plate. For $\alpha_{0} \neq 0$ only one of these two distortions is stable and the equilibrium surface torque has a well defined sign.

In the case of the geometry $i=1$ (or $i=3$ ) (Fig. 3a) we must consider that the orientation of the director $\mathbf{n}$ depends, by the symmetry of this system, only on the distance $y^{\prime}$ from the glass plate $\left(\mathbf{n}=\mathbf{n}\left(y^{\prime}\right)\right)$. Therefore we can assume the director $\mathbf{n}$ lies in the $x^{\prime}, y^{\prime}$ plane i.e. $\mathbf{n}=\left(n_{x}^{\prime}, n_{y}^{\prime}, 0\right)=(\sin \delta, \cos \delta, 0)$, where $\delta$ is the angle between the director and the $y^{\prime}$-axis. The total free energy per unit surface is

$$
\begin{aligned}
J \equiv \int_{-\infty}^{0}\{ & \frac{1}{2} K_{33}\left(1+\eta \sin ^{2} \delta\right)\left(\frac{\partial \delta}{\partial y^{\prime}}\right)^{2}- \\
& \left.-\frac{\chi_{\alpha}}{2} H^{2} \sin ^{2}(\alpha+\delta)\right\} \mathrm{d} y^{\prime}+W\left(\varphi_{0}\right)
\end{aligned}
$$

where $\eta=\frac{K_{11}-K_{33}}{K_{33}}$ is the anisotropy of the elastic constants, $\chi_{\alpha}$ is the diamagnetic anisotropy, $K_{33}$ is the bend elastic constant, $W\left(\varphi_{0}\right)=W_{0} \sin ^{2} \varphi_{0}$ is the anchoring energy function, $\varphi_{0}$ is the angle between the director at the surface and the easy axis and $\alpha$ is the angle between the unitary vector $\mathbf{k}$ orthogonal to the glass plate and the $y$-axis orthogonal to the magnetic field.

From equation (8) one obtains the Euler-Lagrange equation (9) for $\varphi\left(y^{\prime}\right)$ with the boudary condition (10)

$$
K_{33}\left(1+\eta \sin ^{2} \delta\right)\left(\frac{\partial}{\partial y^{\prime}} \delta\right)^{2}-\chi_{\alpha} H^{2} \cos ^{2}(\alpha+\delta)=0
$$

$\left.K_{33}\left(1+\eta \sin ^{2} \delta\right) \frac{\partial \delta}{\partial y^{\prime}}\right|_{y^{\prime}=0}=W_{0} \sin 2 \varphi_{0}$.

From equation (9) we can deduce $\partial \delta / \partial y^{\prime}$ :

$$
\frac{\partial \delta}{\partial y^{\prime}}= \pm \sqrt{\frac{\chi_{\alpha}}{K_{33}}} \frac{H \cos (\alpha+\delta)}{\sqrt{1+\eta \sin ^{2} \delta}}
$$

which can be substituted into equation (10) to give the boundary condition

$$
\begin{array}{r}
\mp \sqrt{K_{33} \chi_{\alpha}}\left(1+\eta \sin ^{2} \varphi\right)^{1 / 2} H \cos (\alpha+\varphi)= \\
=-W_{0} \sin 2 \varphi_{0}
\end{array}
$$

where we have defined the surface director angle $\varphi=\delta(0)$ (see Fig. 3a). By substituting equation (11) into equation (3) one obtains (for $\eta<0$ ) :

$$
\begin{aligned}
\frac{\tau}{S}= \pm \frac{\sqrt{K_{33} \chi_{\alpha}}}{2} H\{-\cos (\alpha+\varphi)(1 & \left.+\eta \sin ^{2} \varphi\right)^{1 / 2}+ \\
& +\frac{1+\eta}{\sqrt{-\eta}} \cos \alpha\left[ \pm \operatorname{arcsinh}\left(\frac{\sqrt{-\eta} \sin \alpha}{\sqrt{1+\eta}}\right)-\operatorname{arcsinh}\left(\frac{\sqrt{-\eta} \cos \varphi}{\sqrt{1+\eta}}\right)\right] \\
& \left.+\frac{\sin \alpha}{\sqrt{-\eta}}[\arcsin (\sqrt{-\eta} \sin \varphi) \mp \arcsin (\sqrt{-\eta} \cos \alpha)]\right\}
\end{aligned}
$$

where $S$ is the total area of the two planes surfaces of the glass plate $P$ of figure 1 . The signs $\mp$ and \pm in equations (12) and (13) stand for the two cases $0<$ $\alpha+\varphi<180^{\circ}$ and $-180^{\circ}<\alpha+\varphi<0$, respectively. $\varphi=90^{\circ}$ in equations (12) and (13) corresponds to the geometry $i=1$ (homogeneous alignment), whilst $\varphi=0^{\circ}$ corresponds to the geometry $i=3$ (homeotropic alignment).

In the case of low magnetic fields $\sqrt{K_{i i} X_{\alpha}} \times$ $\left.H \cos (\alpha) \ll W_{0}\right)$, the angle of the director at the interface is not modified appreciably by the magnetic field and thus, the surface torque is a linear function of the magnetic field (see Eq. (6)) to Eq. (13)). The measurement of $\tau$ for the three geometries of figure 2 allows to obtain the elastic constants $K_{11}, K_{22}$ and $K_{33}$. In particular $K_{22}$ is obtained by measuring the surface torque which corresponds to the geometry $i=2$
(Eq. (6)). The procedure to obtain $K_{11}$ and $K_{33}$ is more complex since, in this case (Eq. (13)), the surface torque is a function of both $K_{33}$ and $\eta$. $\eta$ can be obtained by measuring the ratio of the torque $\tau(i=1)$ to the torque $\tau(i=3)$. In fact this ratio is a function of $\eta$ only. Once $\eta$ is measured, $K_{33}$ can be obtained by substituting the $\eta$-value in the theoretical expression of $\tau(i=1)$ or of $\tau(i=3)$. This procedure gives large errors on $\eta$. In order to clarify this point we make a power expansion of the surface torque of equation (13) in terms of the $\eta$ parameter for the two cases $i=1$ $\left(\varphi=-90^{\circ}, \alpha=90^{\circ}\right)$ and $i=3\left(\varphi=0^{\circ}, \alpha=0^{\circ}\right)$.

After a straightforward calculation we obtain

$$
\frac{\tau}{S}(i=1) \simeq \mp \sqrt{K_{33} \chi_{\alpha}}\left(1+\frac{\eta}{6}\right) H
$$


and

$$
\frac{\tau}{S}(i=3) \simeq \mp \sqrt{K_{33} \chi_{\alpha}}\left(1+\frac{\eta}{3}\right) H
$$

Therefore the relative difference of these surface torques is

$$
\Delta=\frac{\tau(i=3)-\tau(i=1)}{\tau(i=1)} \simeq \frac{\eta}{6}
$$

which is a small value since $\eta$ is usually small. For example, in the case of the nematic LC 5CB the experimental value of $\eta$ is $-0.24(1)$ and thus, $\Delta \sim-4 \%$. As a consequence of this a $5 \%$ uncertainty on the measurement of the surface torques $\tau(i=1)$ and $\tau(i=3)$ can give errors greater than $200 \%$ on $\eta$. Therefore the torque measurements do not give accurate values of the splay elastic constant $K_{11}$.

The values of $K_{33}$ and $\eta$ could be also obtained from the dependence of the torque on the angle $\alpha$ for a given experimental arrangement $(i=1$ or $i=3)$. This latter procedure gives, in way of principle, more accurate results but it has not been used in the present experiment because of the presence of some spurious effects which will be discussed in section 3.1.

So far we have assumed that the magnetic field is small and it does not modify appreciably the surface director angle $\left(\varphi_{0} \sim 0\right)$. In this case the elastic torque is a linear function of the magnetic field. However, if the magnetic field is increased enough, it can modify the surface director angle. Therefore the elastic torque is no yet a linear function of $H$ (see Eq. (6) to Eq. (13)). By looking at equation (6) to equation (13) we find that this occurs when $H \gtrsim \frac{2 W_{0}}{\sqrt{\chi_{\alpha} K_{i i}} \cos \alpha}$. This latter condition is accomplished when the magnetic coherence length $\xi=\sqrt{\frac{K_{i i}}{\chi_{\alpha}}} \frac{1}{H}$ becomes comparable to the extrapolation length $\delta \simeq \frac{K_{i i}}{W_{0}}$. If the non linear regime is reached both $K_{i i}$ and $W_{0}$ can be obtained in a unambiguous way by the best fit of the experimental dependence of the surface torque on the magnetic field (see Eqs. (6) to (13)). Therefore the surface torque measurements allow to obtain, in principle, both the elastic constants and the anchoring energy coefficient. Evidence for large deviations of $\tau / S$ from a linear behaviour has been reported by us in a recent paper(23) where the azimuthal anchoring energy coefficient of the SiO-nematic interface was measured. The accuracy of the anchoring energy measurements depends greatly on the values of the angles $\alpha_{0}$ and $\alpha$. Consider, for instance, the twist case (Eqs. (6) and (7)). The surface torque depends on the director angle at the surface through a cosine function which is much more sensitive to variations of the surface director angle $\varphi$ when $\alpha \simeq 90^{\circ}$. Therefore the measurements of anchoring energy are more accurate if $\alpha_{0}$ is close to $90^{\circ}$, whilst the measurements of elastic constants are more accurate if $\alpha_{0}$ is small enough.

The main advantages and drawbacks of the torque measurements with respect to the "standard " Freedericksz transition measurements are :

Advantages : 1) torque measurements are poorly sensitive to small misalignments of the director at the surface if the angle between the magnetic field and the director is close to $90^{\circ}$. Under these conditions, for example, a $2^{\circ}$ uncertainty on the director orientation gives a relative error on the torque lower than $0.1 \%$. This conclusion holds also if the director misalignment is not the same all over at the interface. This can be easily understood if one looks at equation (3) which gives the surface torque $\tau$. This equation shows that the total surface torque comes from the superposition of the contributions of the magnetic torques acting on the different regions of the nematic sample. In particular the largest contribution to the magnetic torque comes from these regions of the sample where the angle $\theta$ between the director and the magnetic field is $\sim 45^{\circ}$, whilst the minor contribution comes from the regions where $\theta \sim 90^{\circ}$ or $\Theta=0^{\circ}$. Therefore, if the surface angle is close to $90^{\circ}$, the total torque $\tau$ is poorly sensitive to details of the surface arrangement. 2) A weak anchoring energy can be evidenced, in a unambiguous way, by looking at the linearity of the magnetic field-dependence of the surface torque. The best fit of the experimental results allows to obtain both the elastic constants and the anchoring energy coefficient. Therefore a weak anchoring of the director at the interfaces does not affect the accuracy of measurements.

Drawbacks : 1) A large amount of nematic sample need to perform this kind of measurements $\left(\mathrm{a} \mathrm{few} \mathrm{cm}^{3}\right.$ ), 2) An uniform orientation of the director is required on a large surface region $\left(\sim 3 \mathrm{~cm}^{2}\right)$. 3) The measurement of the $K_{11}$ elastic constant by means of the torque technique is affected by a large uncertainty. This latter is just the most important drawback of the present technique.

The main error sources of the torque technique are : a) the inaccuracy of the area $S$ of the glass plate $(\sim 1 \%)$. b) The inaccuracy of the torsion coefficient of the quartz wire $W(\sim 0.5 \%)$. c) The inaccuracy of the rotation $\Delta \alpha$ of the torsion wire $\left(\sim 10^{-4} \mathrm{rad}\right)$. This gives a relative error on the torque $\tau$ of about $1 \%$ in the present experiment. d) The inaccuracy due to the presence of a spurious background ( $\sim 1-2 \%)$. This contribution will be discussed in section 3.1. e) The inaccuracy of the magnetic field $(\sim 0.5 \%)$. f) The inaccuracy due to the boundary effects which have been neglected in order to obtain equations (6) to (13). The maximum contribution due to this source of error is expected when the director on the small lateral surface of the glass plate is oriented along the same easy axis of the two plane surfaces of the glass plate. In this case a relative contribution to the torque of the order of $\Delta S / S$ is expected, where $\Delta S$ represents 
the total area of the lateral surface. In the present experiment $\frac{\Delta S}{S}<1.5 \%$. By considering all these error sources we estimate a maximum relative error on the parameter $A=\frac{\tau}{S . H}$ of about $5 \%$. The corresponding uncertainty of $K_{33}$ and $K_{11}$ are estimated to be $20 \%$ and $50 \%$, respectively. These large errors come from the large uncertainty of $\eta$. The accuracy of $K_{33}$ can be increased to $10 \%$ if the $\eta$-parameter is known from independent measurements. In this case $K_{33}$ can be obtained from measurements performed either in the geometry $i=1$ or in the geometry $i=3$. Notice that the uncertainty of $\eta$ poorly affects the accuracy of $K_{33}$ particularly in the case of the geometry $i=1$ (see Eq. (14)).

\section{Experimental results.}

3.1 EXPERIMENTAL PROCEDURES. - In this section we report some experimental results concerning the geometries $i=1$ and $i=3$ of figure 2. Experimental results for the twisted geometry $(i=2)$ have already been reported in a previous paper [17]. The nematic sample is 4-pentyl-4'-cyanobiphenyl (5CB) produced by $\mathrm{BDH}$ which has the clearing temperature $T_{\mathrm{c}}=$ $35.3^{\circ} \mathrm{C}$. The homeotropic alignment on the glass plate $(i=3)$ is obtained by dipping the glass plate in a $10 \%$ water-solution of alkyl benzene sulfonate (RBS) at the temperature $T=70^{\circ} \mathrm{C}$ in a ultrasonic bath. The homogeneous orientation in the plane of the glass plate $(i=1)$ is obtained by oblique evaporation of $\mathrm{SiO}$ at a $60^{\circ}$-incidence angle [24] on both the plane surfaces of the glass plate. This latter technique gives an uniform director alignment on the whole plate with a high anchoring energy. In order to check the director alignment at the surfaces of the glass plate we make a thin nematic layer by sandwiching the nematic between two parallel glass plates treated in the same way. The orientation of the director in this layer is observed by using a Zeiss polarizing microscope. Both the surfaces of a single glass plate are analysed in such a way, After, the glass plate is suspended to the quartz wire and dipped in the nematic sample, and the torque $\tau$ is measured (Fig. 1).

A great care is devoted to reduce spurious magnetic torques on the torsion pendulum. These spurious torques can be due to small residual gradients of the magnetic field, to capillarity effects and to the anisotropic shape of the glass plates $\mathbf{P}$ and $\mathbf{M}$ of figure 1 . For a diamagnetic plate these effects are expected to depend on the square power of the magnetic field. The spurious effects are evaluated by measuring the residual torque exerted on the glass plate when the nematic LC is in its isotropic phase. In our experiment the spurious torque depends on $H$ in a much more complex way than a simple quadratic form. This suggests the presence of a small amount of ferroma- gnetic impurities in the experimental apparatus. This torque depends on the angle between the glass plate $P$ and the magnetic field. A suitable choice of this angle allows to reduce the spurious torque to less than $5 \%$ with respect to the torque measured in the anisotropic phase. Furthermore the spurious torque does not change (within our experimental accuracy) when the temperature is increased by more than $15^{\circ} \mathrm{C}$ above the clearing value. Therefore we can correct the torque measured in the anisotropic phase of the nematic liquid crystal by subtracting the spurious background measured in the isotropic phase. The reliability of this latter procedure is checked by looking at the magnetic field-dependence of the torque in the anisotropic phase. In fact, uncorrected experimental values show some small (1-2\%) systematic and not monotonic deviations from linearity which disappear after correction for the spurious background. Under this conditions, we estimates that the presence of the spurious torque gives a residual error on the experimental measurements lower than $1-2 \%$.

However the presence of these spurious effects forces us to perform the experiment by orienting the glass plate at the angle $\alpha$ which minimizes the spurious torque. As a consequence of this the dependence of the surface torque on the angle cannot be investigated in detail. This limits greatly the accuracy of the measurement of the splay elastic constant as we have already shown (Sect. 2).

Accurate and reproducible measurements of elastic constants need an uniform director distortion occurring in the nematic sample. This condition can be accomplished by cooling the LC starting from the isotropic phase in the presence of a high magnetic field $(8.8 \mathrm{kG})$ which makes an angle $\beta \neq 90$ with the easy axis of the director. Figure 4 shows the surface torque measured at the temperature $T=30^{\circ} \mathrm{C}$ versus the $\beta$-angle in the geometry $i=1$. Each experimental value of $\tau$ in figure 4 has been obtained after having cooled the sample from the isotropic phase in the presence of the $8.8 \mathrm{kG}$ magnetic field. According to the theory (Eq. (11)) the surface torque vanishes when $\beta \sim 90^{\circ}$. An analogous dependence of $\tau$ on the $\beta$-angle is found for the geometry $i=3$ of figure 2 .

A different behaviour occurs if the magnetic field is swiched on when the nematic sample is in the anisotropic phase. In this case the measured value of the torque is much smaller than the corresponding one obtained by the previous procedure. This behaviour indicates that the director distortion close to the glass plate is not uniform and domains with opposite distortions of the director-field occur [22]. If $\beta \neq 90^{\circ}$, the two states characterized by opposite distortions of the director-field have different free energies. Therefore the distortion which corresponds to a higher value of the free energy is unstable and tends to relax with time [22]. In order to measure the relaxation time from the unstable state to the stable one we have 


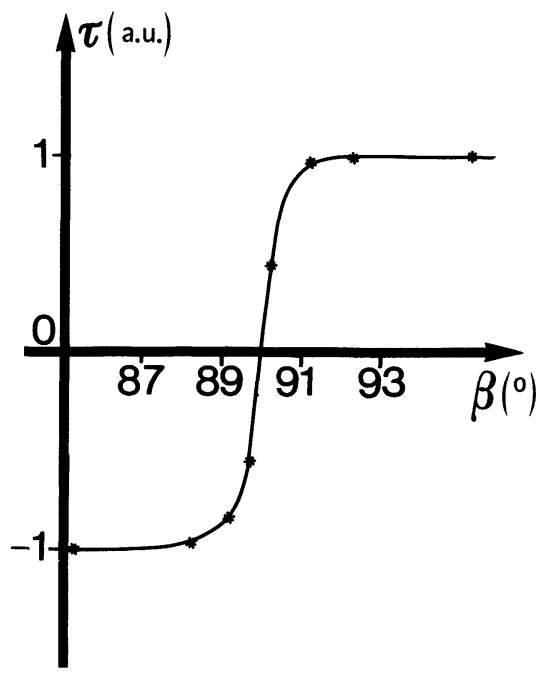

Fig. 4. - Dependence of the elastic surface torque on the angle $\beta$ between the easy axis and the magnetic field in the geometry $i=1$ (homogeneous director orientation). The torque is expressed in arbitrary units. Each experimental point has been obtained after having cooled the nematic sample from the isotropic phase in the presence of a $8.8 \mathrm{kG}$ magnetic field. The temperature of the sample is $T=30^{\circ} \mathrm{C}$.

prepared an uniformly distorted sample by cooling the LC from the isotropic phase in the presence of the $8.8 \mathrm{kG}$ magnetic field for $\beta=70^{\circ}$. Once the sample had reached the temperature $T=30^{\circ} \mathrm{C}$, we rotated the torsion pendulum to obtain a $\beta$-angle greater than $90^{\circ}$. In this condition the original distortion is not stable and must relax to the stable one. The relaxation of the system can be detected by measuring the timedependence of the surface torque. Figure 5 shows some relaxation curves obtained by this procedure for different values of the $\beta$-angle. The geometry of the glass plate corresponds to the $i=1$ case of figure 2 (homogeneous alignment). As expected the free energy difference between the two director distortions is an increasing function of $\beta-90^{\circ}$, and thus, the relaxation time $T_{\mathrm{R}}$ (see Fig. 5) becomes slower and slower as $\beta$ approaches $90^{\circ}$. In particular we find that $T_{\mathrm{R}}$ diverges (slowing down) when a critical angle $\beta_{\mathrm{c}} \sim 93^{\circ}$ is reached. For $\beta \geqslant \beta_{\mathrm{c}}$ the relaxation rate $\lambda_{\mathrm{R}}=1 T_{\mathrm{R}}$ shows a critical behaviour $\left[\lambda_{\mathbb{R}} \propto\left(\beta-\beta_{c}\right)\right]$. For $90^{\circ}<\beta<\beta_{\mathrm{c}}$ no relaxation is observed.

In the other experimental geometries (cases $i=3$ and $i=2$ ), the relaxation from the unstable state does not occur in the whole range of allowable $\beta$-angles $\left(70^{\circ}<\beta<110^{\circ}\right)$. In particular no change of the surface torque was observed after one day. This suggests that the critical angle $\beta_{\mathrm{c}}$, in these two latter cases, is greater than $110^{\circ}$.

3.2 Elastic CONSTANT MEASUREMENTS. - As shown in section 2 , the torque measurements furnish the product $K_{33} \chi_{\alpha *}$. Therefore the effective accuracy

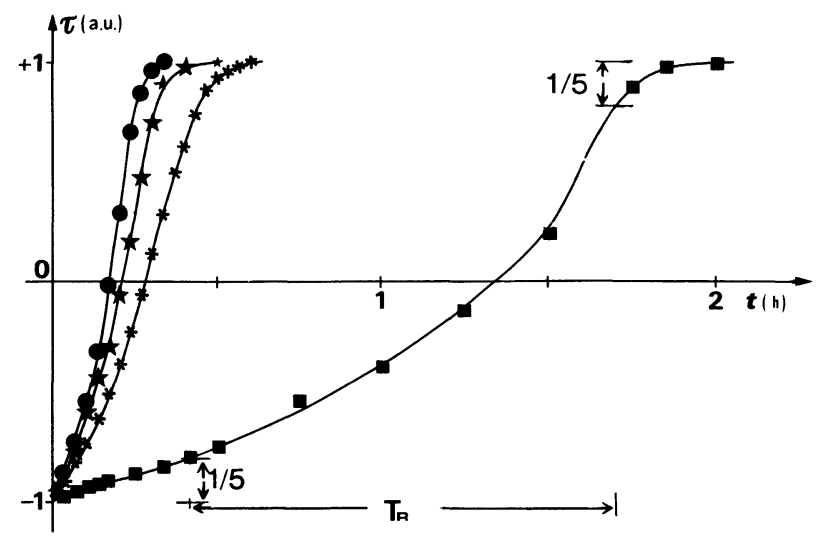

Fig. 5. - Time relaxation from an unstable state to a stable one for different values of the $\beta$-angle between the easy axis and the magnetic field : (๑) $\beta=98.25^{\circ}$, ( $\left.\star \star\right) ~ \beta=$ $96.75^{\circ},(*) \beta=95.25^{\circ},(\boldsymbol{\square}) \beta=93.25^{\circ}$. The measurements are performed in the geometry $i=1$ of figure 2 . On the ordinate scale is reported the measured value of the torque expressed in arbitrary units. For convenience the experimental torques corresponding to different values of the $\beta$-angle have been rescaled in such a way as to coincide at the time $t=0$. $T_{\mathrm{R}}$ represents the relaxation time. The magnetic field value is $8.8 \mathrm{kG}$ and the temperature of the nematic sample is $T=30^{\circ} \mathrm{C}$.

of the elastic constants depends also on the accuracy of the diamagnetic anisotropy $\chi_{\alpha}$. Analogously in the case of the Freedericksz transition the measurements furnish the ratio $K_{33} / \chi_{\alpha^{\circ}}$. Measurements of the diamagnetic anisotropy are often affected by large experimental inaccuracies. Therefore a comparison between the experimental results obtained by Freedericksz transition experiments and by torque measurements can furnish some indications on the reliability of the allowable values of $\chi_{\alpha}$. In the case of 5CB two different measurements of $\chi_{\alpha}$ have been reported [25, 26]. The values of $\chi_{\alpha}$ given in reference [25] are $\sim 8 \%$ higher than those of reference [26]. The elastic constants of 5CB have been measured by Madhusudana and Pratibha [1] and by Bunning et al. [27] who used the Freedericksz transition technique. The threshold fields measured in these papers agree between them within a few per cent. Therefore, in the following we will refer only to the results of reference [27]. The authors of reference [27] obtained the elastic constants by using the $\chi_{\alpha}$-values given in reference [26]. Different values of the elastic constants can be obtained by using the same results of reference [27] but the magnetic anisotropy given in reference [25]. In the following we will denote these two sets of elastic constants and corresponding diamagnetic anisotropies as " DATA $I$ » $[26$, 27] and "DATA II " [25, 27], respectively, These parameters are reported in table I.

The elastic torque per unit surface versus the magnetic field in the geometry $i=1$ is shown in figure 6 . Different symbols correspond to different temperatures. The linear dependence of $\tau$ on the magnetic field ensures that the anchoring energy coefficient is 
Table I. - Splay and bend elastic constants and diamagnetic anisotropies of 5CB. Data I and Data II correspond to the elastic constants calculated by using the experimental Freedericksz threshold fields given in reference [27] and the diamagnetic anisotropies given in references [26] and [25]. Our results indicated with and without the apostrophe have been obtained by using the torques measured in the present experiment and the diamagnetic anisotropies given in references [25] and [26].

\begin{tabular}{|c|c|c|c|c|c|c|c|c|c|}
\hline \multirow{4}{*}{ DATA I } & $T_{\mathrm{c}}-T\left({ }^{\circ} \mathrm{C}\right)$ & 13.4 & 10.4 & 8.4 & 6.0 & 3.8 & 2.2 & 1.2 & 0.3 \\
\hline & $\chi_{\alpha}\left(\times 10^{-7}\right)$ & 1.11 & 1.07 & 1.04 & 0.99 & 0.93 & 0.86 & 0.80 & 0.70 \\
\hline & $K_{11}\left(\times 10^{-7}\right.$ dyne $)$ & 6.45 & 5.84 & 5.40 & 4.78 & 4.13 & 3.53 & 2.91 & 2.07 \\
\hline & $K_{33}\left(\times 10^{-7}\right.$ dyne $)$ & 8.82 & 7.79 & 7.00 & 6.03 & 5.00 & 4.13 & 3.43 & 2.18 \\
\hline \multirow{3}{*}{ DATA II } & $\chi_{\alpha}^{\prime}\left(\times 10^{-7}\right)$ & 1.19 & 1.16 & 1.13 & 1.08 & 1.01 & 0.94 & 0.87 & 0.79 \\
\hline & $K_{11}^{\prime}\left(\times 10^{-7}\right.$ dyne $)$ & 6.95 & 6.34 & 5.88 & 5.20 & 4.49 & 3.84 & 3.18 & 2.34 \\
\hline & $K_{33}^{\prime}\left(\times 10^{-7}\right.$ dyne $)$ & 9.49 & 8.46 & 7.62 & 6.56 & 5.44 & 4.48 & 3.75 & 2.47 \\
\hline \multirow{4}{*}{$\begin{array}{c}\text { OUR } \\
\text { RESULTS }\end{array}$} & $K_{11}\left(\times 10^{-7}\right.$ dyne $)$ & 9.00 & 7.83 & 6.85 & 5.79 & 4.55 & 4.19 & 3.55 & 2.69 \\
\hline & $K_{33}\left(\times 10^{-7}\right.$ dyne $)$ & 8.61 & 7.67 & 7.20 & 6.36 & 5.52 & 4.47 & 3.67 & 2.48 \\
\hline & $K_{11}^{\prime}\left(\times 10^{-7}\right.$ dyne $)$ & 8.39 & 7.22 & 6.31 & 5.31 & 4.19 & 3.83 & 3.26 & 2.39 \\
\hline & $K_{33}^{\prime}\left(\times 10^{-7}\right.$ dyne $)$ & 8.03 & 7.07 & 6.63 & 5.83 & 5.08 & 4.09 & 3.37 & 2.20 \\
\hline
\end{tabular}

strong enough. Since the relative accuracy on the measurement of $\tau$ is $\sim 2 \%$, we estimate from equations (12) and (13) an anchoring energy coefficient greater than $2 \times 10^{-3} \mathrm{erg} / \mathrm{cm}^{2}$ in the whole range of nematicity of $5 \mathrm{CB}$. This result is consistent with the value $W_{0} \sim 2 \times 10^{-2} \mathrm{erg} / \mathrm{cm}^{2}$ reported in reference [28]. The parameter $A(1)=\frac{\tau(i=1)}{S H}$ can be obtained by the best linear fit of the experimental results of figure 6 . The temperature dependence of $A(1)$ is shown in figure 7 . The full and broken curves in figure 7 correspond to the theoretical torques calculated by substituting in equation (13) the sets of data I and II, respectively.

The elastic torque per unit surface versus the magnetic field $H$ in the case $i=3$ is shown in figure 8 . In this case, too, the linear dependence of $\tau$ on the magnetic field indicates the anchoring of the director at the surface is strong enough. In particular we can estimate that the anchoring energy coefficient for the homeotropic alignment due to RBS is greater than $5 \times 10^{-3} \mathrm{erg} / \mathrm{cm}$. The best linear fit of the experimental results of figure 8 allows us to obtain the parameter $A(3)=\frac{\tau(i=3)}{S H}$. The temperature dependence of $A(3)$ is shown in figure 9. The full and broken lines correspond to the theoretical values obtained by substituting in equation (13) the two sets of data I and II, respectively. Notice that in both the geometries $i=1$ and $i=3$ the measured torques lie between the full and broken curves. Therefore our results agree with those of reference [27] if one accounts for the uncertainty on the diamagnetic anisotropy. Furthermore our results suggest that the correct value of the diamagnetic anisotropy is intermediate between those given in references [25] and [26].

The experimental values of $\eta$ and $K_{33}$ can be obtained from $A(1)$ and $A(3)$ by using the procedure already discussed in section 2 . The values of $K_{11}$ and $K_{33}$ obtained in this way are reported in table $I$. The elastic constants with and without the apostrophe have been obtained by using the diamagnetic anisotropies given in references [25] and [26], respectively. As shown in section 2, the accuracy of $K_{11}$ is poor ( $\sim 50 \%$ ), whilst the accuracy of $K_{33}$ is estimated of the order of $20 \%$.

More accurate values of $K_{33}(\sim 10 \%)$ are obtained by using equation (13) together with the values of $\eta$ measured by other methods [27]. The bend elastic constant $K_{33}$ can be obtained by substituting in equation (13) the experimental values of either $A(1)$ or $A(3)$. Comparison between the values of $K_{33}$ obtained from these two independent measurements gives a direct check of the accuracy of our experimental results. Figure 10a shows our experimental values of $K_{33}$ versus the temperature as deduced from $A(1)$. Full circles and open circles correspond to the values of $K_{33}$ obtained by substituting in equation (13) 


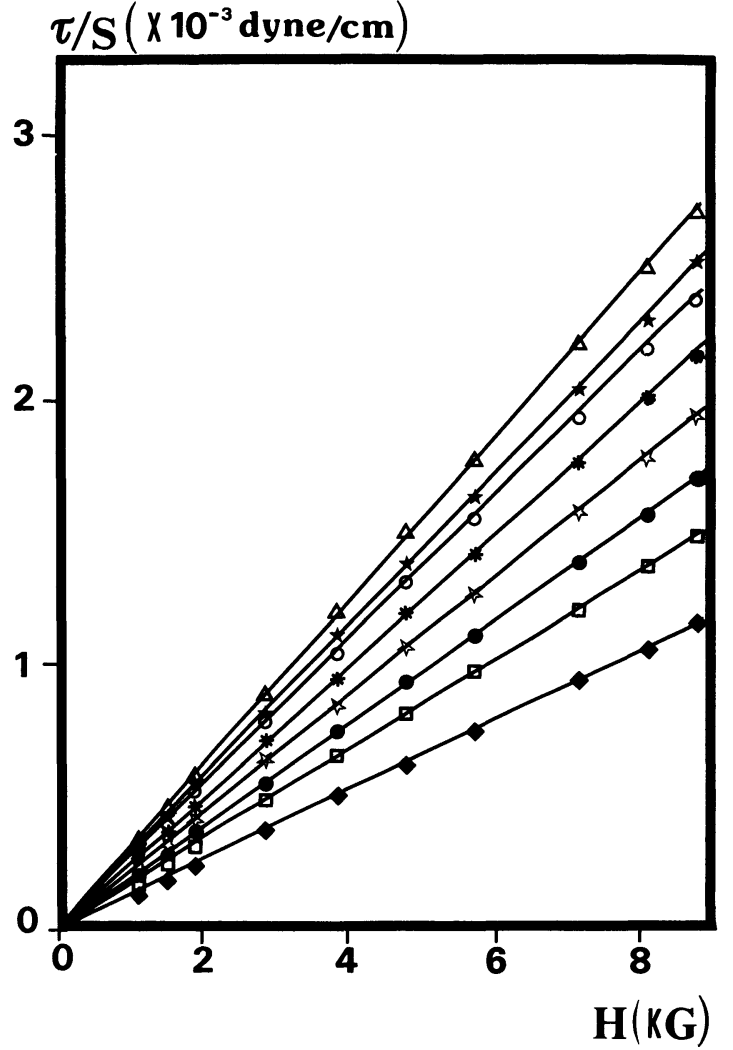

Fig. 6. - Torque for unit surface area in the case $i=1$ $\left(\varphi=-90^{\circ}\right)$ versus the magnetic field. Different symbols correspond to different values of the temperature : $(\Delta)$ $T_{\mathrm{c}}-T=13.4{ }^{\circ} \mathrm{C},(\star) T_{\mathrm{c}}-T=10.4{ }^{\circ} \mathrm{C},(\mathrm{O}) T_{\mathrm{c}}-T=$ $8.4^{\circ} \mathrm{C},(*) T_{\mathrm{c}}-T=6{ }^{\circ} \mathrm{C}$, (古) $T_{\mathrm{c}}-T=3.8^{\circ} \mathrm{C}$, (ө) $T_{\mathrm{c}}-$ $T=2.2^{\circ} \mathrm{C},(\square) T_{\mathrm{c}}-T=1.2{ }^{\circ} \mathrm{C},(\square) T_{\mathrm{c}}-T=0.28^{\circ} \mathrm{C}$ The full lines represent the best linear fit of the experimental results. The value of the $\alpha$-angle between the $y$-axis and the axis orthogonal to the glass plate is $87.3^{\circ}$ (see Fig. 3).

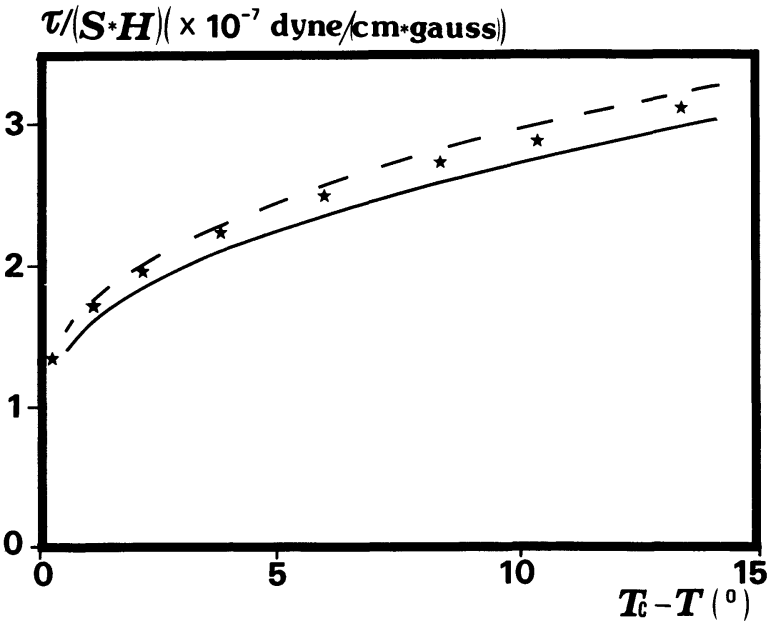

Fig. 7. - Temperature dependence of $A(1)=\frac{\tau(i=1)}{S H}$.

The full and broken curves correspond to the expected values of $A(1)$ as obtained by substituting in equation (11) the two sets of experimental data denoted as I and II, respectively (see discussion in the text). The value of the angle $\alpha$ is $\alpha=87.3^{\circ}$.

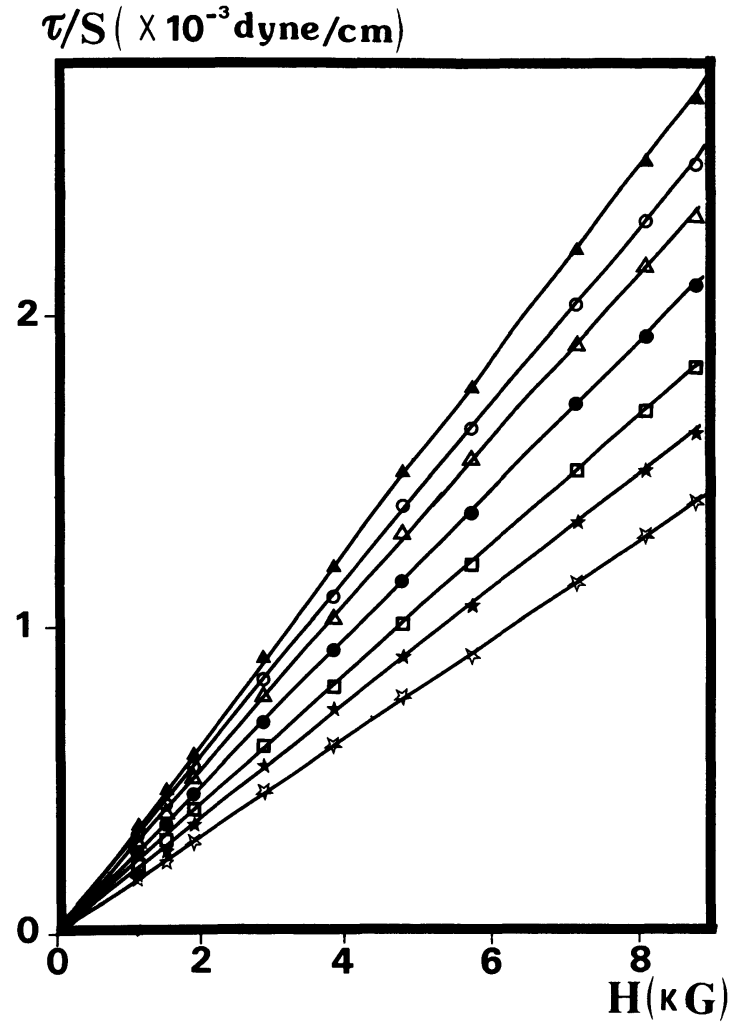

Fig. 8. - Elastic torque per unit surface area versus the magnetic field in the case $i=3\left(\varphi=0^{\circ}\right)$ for different values

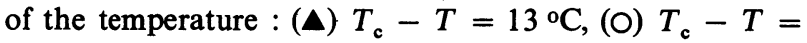
$9.9^{\circ} \mathrm{C},(\triangle) T_{\mathrm{c}}-T=8{ }^{\circ} \mathrm{C},(\circlearrowleft) T_{\mathrm{c}}-T=5.5^{\circ} \mathrm{C}$, ( $\left.\square\right)$

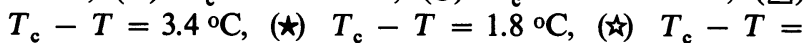
$0.8^{\circ} \mathrm{C}$. The full lines correspond to the best linear fit of the experimental results. The value of the $\alpha$-angle is $10.6^{\circ}$.

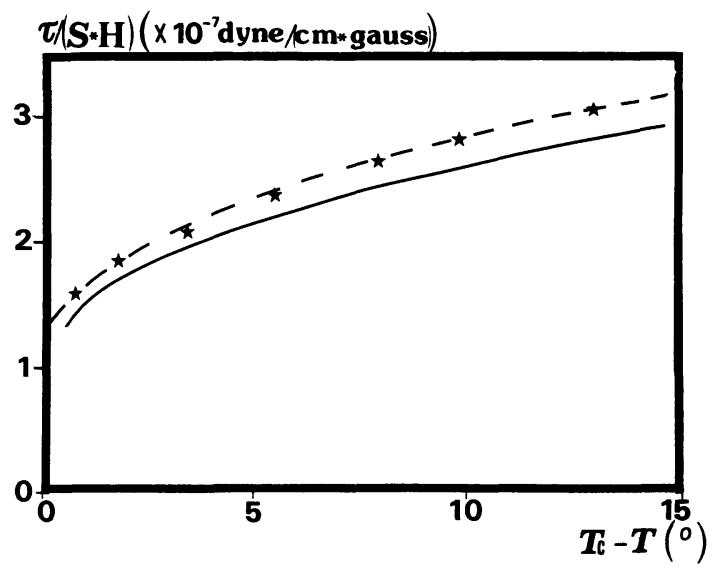

Fig. 9. - Temperature dependence of $A(3)=\frac{\tau(i=3)}{S H}$. The full and broken curves correspond to the theoretical torques calculated by using the same procedure described in figure 7. The value of the angle $\alpha$ is $10.6^{\circ}$.

the $\chi_{\alpha}$ values given in references [26] and [25], respectively. The full and broken curves correspond to the elastic constants $K_{33}$ denoted as data I and II, respectively. Figure 10b shows the values of $K_{33}$ obtained by using the experimental values of $A(3)$. 


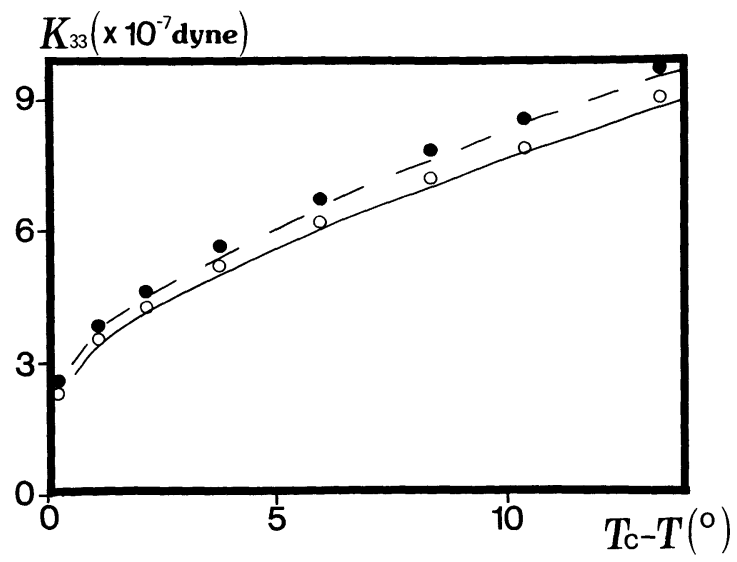

a)

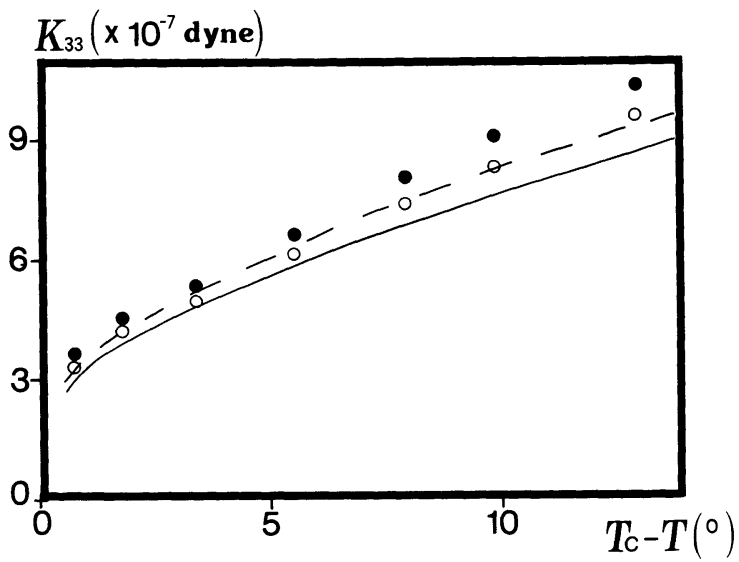

b)

Fig. 10. - a) Experimental values of $K_{33}$ as obtained from surface torque measurements in the case of the geometry $i=1$ of figure 2. b) Experimental values of $K_{33}$ obtained from torque measurements in the geometry $i=3$. Full circles and open circles represent our experimental values of $K_{33}$ obtained by using the values of $\chi_{\alpha}$ given in references [26] and [25]. The full and broken lines correspond to the experimental values of $K_{33}$ denoted as data I and data II in table I, respectively.

\section{Conclusions.}

Experimental measurements of the torque exerted by a nematic LC on an interface are shown to furnish a new method to measure the elastic constants of nematic LC. In the case of weak anchoring of the director at the interfaces, the anchoring energy too can be obtained in an unambiguous way from surface torque measurements. Note that the torque method can also be used to measure the anchoring energy when the director alignment at the interface is tilted. A direct measurement of the anchoring energy coefficient at a SiO-nematic interface obtained by using the elastic torque method has been given by us in reference [23]. The torque measurements are practically unaffected from the typical error sources affecting the "standard " Freedericksz transition method (director misalignment at the surfaces, weak anchoring energy). However, the torque measurements are more complex and the splay elastic constant cannot be actually obtained with a satisfactory accuracy. A great improvement of the torque method could be obtained if the spurious angle-dependent background was suitably reduced. In this case both the bend and splay elastic constants could be obtained with a better accuracy from measurements of the angular dependence of the elastic torque in a single experimental geometry ( $i=1$ or $i=3$ in Fig. 2).

In our opinion the torque technique finds its best application in the measurement of the twist elastic constant $K_{22}$ of nematic LC. In fact the optical measurements of $K_{22}$ from the Freedericksz threshold are particularly difficult and affected by large experimental errors [1]. On the contrary the measurements of the twist elastic constant by the torsion pendulum are the simplest ones since they concern a single experimental geometry ( $i=2$ in Fig. 2) [17].

An open question concerns the role played by the surface-like elastic constant introduced by Nehring and Saupe [20]. The satisfactory agreement between our experimental results and the theoretical predictions obtained by assuming $K_{13}=0$ (Figs. 7 and 9) seem to indicate that $K_{13}$ does not play an important role when the geometries of figure 2 are investigated. However a definitive response to this question cannot be reached before a full theory of the surface elasticity is developed [21].

In conclusion, although the present technique cannot replace the Freedericksz transition method, it can constitute a useful alternative way to measure the twist and bend elastic constants and the anchoring energy of liquid crystals. Furthermore, a great increase of the experimental accuracy of this method can be expected if some technical difficulties will be overcomed in the future work.

\section{References}

[1] Madhusudana, N. V. and Pratibha, R., Mol. Cryst. Liq. Cryst. 89 (1982) 249.

[2] Schad, Hp. and Osman, M. A., J. Chem. Phys. 75 (1981) 880 .

[3] De Jeu, W. H., Claassen, W. A. P. and Spruit, A. M. J., Mol. Cryst. Liq. Cryst. 37 (1976) 277.
[4] Haller, I., J. Chem. Phys. 57 (1972) 1400.

[5] LeEnhouts, F., Von Der Wounde, F. and DeKKer, A. J., Phys. Lett. 58A (1976) 242.

[6] Oldano, C., Miraldi, E., Taverna Valabrega, P., J. Physique 45 (1984) 755 and

Oldano, C., Miraldi, E., Strigazzi, A., Taverna- 
Valabrega, P., Trossi, I., J. Physique 45 (1984) 355.

[7] Maze, C., Mol. Cryst. Liq. Cryst. 48 (1978) 273.

[8] Malvano, R. and Strigazzi, A., Atti. Accad. Sci. Torino 12 (1978) 193, 18 (1979) 1599.

[9] Hiroaki, Usui, Hideo Takezoe, Atsuo Fukuda, and EIICHI, Kuze, Jpn. J. Appl. Phys. 18 (1979) 1599.

[10] Langevin, D. and Bouchiat, H. A., J. Physique Colloq. 36 (1975) C-197.

[11] Miraldi, E., Oldano, C., Taverna Valabrega, P. and Trossi, L., Il Nuovo Cimento 66B (1981) 179.

[12] Skarp, K., Lagerwall, S. T. and Stebler, B., Mol. Cryst. Liq. Cryst. 60 (1980) 215.

[13] Balzarini, D. A., Dunmur, D. A. and PolffyMuhCray, Mol. Cryst. Liq. Cryst. Lett. 102 (1984) 35.

[14] see, for example, DE GenNes, P. G., The Physics of Liquid Crystal (Clarendon Press Oxford) 1974.

[15] Rapini, A. and Papoular, M., J. Physique Colloq. 30 (1969) C4-54.

[16] GrupP, J., Phys. Lett. 99A (1983) 373.

[17] Faetti, S., Gatti, M. and Palleschi, V., J. Physique Lett. 46 (1985) 881.
[18] Byschenkow, G. and Kleman, M., J. Chem. Phys. 64 (1976) 404.

[19] Frank, F. C., Discuss. Faraday Soc. 25 (1958) 19, and Ericksen, J. L., Archs. Nation. Mech. Analysis 23 (1966) 266.

[20] Nehring, J. and Saupe, A., J. Chem. Phys. 56 (1972) 5527 and Nehring, J. and Saupe A., J. Chem. Phys. 54 (1971) 337.

[21] Oldano, C. and Barbero, G., J. Physique Lett. 46 (1985) L-451 and Oldano, C. and Barbero, G., Phys. Lett. 110A (1985) 213.

[22] Leger, L., Mol. Cryst. Liq. Cryst. 24 (1973) 33.

[23] Faetti, S., Gatti, M., Palleschi, V. and Sluckin, T. J., Phys. Rev. Lett. 55 (1985) 1681.

[24] Janning, J. L., Appl. Phys. Lett. 21 (1972) 173.

[25] Scherrel, P. L. and Crellin, D. A., J. Physique Colloq. 40 (1979) C3-213.

[26] Buka, A. and De JeU, W. H., J. Physique 43 (1982) 361.

[27] Bunning, J. D., Faber, T. E. and Sherrel, P. L., J. Physique 42 (1981) 1175.

[28] Yokoyama, H. and Van Sprarg, H. A., J. Appl. Phys. 57 (1985) 4520. 